\title{
Test of theoretical models for ultrafast heterogeneous electron transfer with femtosecond two-photon photoemission data $^{\dagger}$
}

\author{
LARS GUNDLACH ${ }^{1, *}$, TOBIAS LETZIG ${ }^{2}$ and FRANK WILLIG ${ }^{3, *}$ \\ ${ }^{1}$ Department of Chemistry, Rutgers University-Newark, 73 Warren St., Newark, NJ 07102-1811, USA \\ ${ }^{2}$ Johanna Solar Technology, Muenstersche Strasse 24, 14772 Brandenburg, Germany \\ ${ }^{3}$ Fritz Haber Institut der MPG, Faradayweg 4-6, 14195 Berlin, Germany \\ e-mail: larsg $@$ andromeda.rutgers.edu; willig $@$ fhi-berlin.mpg.de
}

\begin{abstract}
The energy distribution of electrons injected into acceptor states on the surface of $\mathrm{TiO}_{2}$ was measured with femtosecond two-photon photoemission. Shape and relative energetic position of these distribution curves with respect to the corresponding donor states, i.e. of perylene chromophores in the first excited singlet state attached via different bridge-anchor groups to the $\mathrm{TiO}_{2}$ surface, were compared with the predictions of different theoretical models for light-induced ultrafast heterogeneous electron transfer (HET). Gerischer's early scenario for light-induced HET was considered and two recent explicit calculations, i.e. a fully quantum mechanical analytical model and a time-dependent density functional theory model based on molecular dynamics simulations for the vibrational modes were also considered. Based on the known vibrational structure in the photoionization spectrum of perylene in the gas phase and that measured in the linear absorption spectra of the perylene chromophores anchored on the $\mathrm{TiO}_{2}$ surface the energy distribution curves for the injected electrons were fitted assuming the excitation of the dominant $0 \cdot 17 \mathrm{eV}$ vibrational mode in the ionized perylene chromophore leading to a corresponding Franck-Condon dictated progression in the energy distribution curves. Each individual peak was fitted with a Voigt profile where the Lorentzian contribution was taken from the time-resolved HET data and the Gaussian contribution attributed to inhomogeneous broadening. The measured room temperature energy distribution curves for the injected electrons are explained with the fully quantum mechanical model for light-induced HET with the high energy, $0.17 \mathrm{eV}$, skeletal stretching mode excited in the ionized perylene chromophore. The corresponding energy distribution of the injected electrons is fully accommodated in acceptor states on the $\mathrm{TiO}_{2}$ surface fulfilling the wide band limit.
\end{abstract}

Keywords. Fully quantum mechanical model; Gerischer model; heterogeneous electron transfer; femtosecond two-photon photoemission; ultrafast dynamics.

\section{Introduction}

Light-induced heterogeneous electron transfer (HET) involves the transfer of an electron from the excited electronic state of a chromophore to the surface of a semiconductor or metal. It is a fundamental process in photoelectrochemistry, ${ }^{1}$ it is utilized in the $\mathrm{AgBr}$ photographic process ${ }^{2}$ and in the dye-sensitized solar cell. ${ }^{3}$ Experimental systems have been investigated that consisted in most cases of chromophores like $\mathrm{Ru}$-bipyridyl with additional ligands ${ }^{3}$ or of aromatic dye molecules like perylene, ${ }^{4}$ in both cases the chromophore was covalently linked to an acidic anchor group, e.g. $-\mathrm{COOH}$, and the latter chemisorbed on the surface of the wide bandgap semiconductor $\mathrm{TiO}_{2}$. Ultrashort injection times of a few

\footnotetext{
Dedicated to the memory of the late Professor S K Rangarajan
} *For correspondence femtoseconds have been reported for such experimental systems when exposed to ultra high vacuum $^{5-7}$ and also to the laboratory ambient. ${ }^{8,9}$ The time scale of the HET process can be shifted to the picosecond range. ${ }^{10,11}$ The general case of lightinduced HET is realized if the ground state of the molecular donor is located in the wide bandgap of a suitable semiconductor and the excited state of the molecular donor above the lower conduction band edge. ${ }^{1}$ In order to preserve monomeric behaviour the perylene chromophores were equipped with two bulky lateral spacer groups. ${ }^{4,6}$ The characteristic vibrational structure of the monomer was indeed maintained in the linear absorption spectra when these modified perylene chromophores, labelled Pe' in the following, were adsorbed on the surface of $\mathrm{TiO}_{2}{ }^{12}$ Ultrashort injection times of a few femtoseconds require that the chromophores are positioned sufficiently close to the surface of the semiconduc- 
tor. In the above cases this was realized by the $-\mathrm{COOH}$ anchor group which inserts the $-\mathrm{C}-\mathrm{O}$-link between the organic chromophore and the $\mathrm{TiO}_{2}$ surface. ${ }^{3,7,13}$ Recently, light-induced HET was timeresolved also on a metal electrode with perylene chromophores attached via the $-\mathrm{CH}_{2}-\mathrm{SH}$ anchor group to the $\operatorname{Ag}(110)$ surface yielding an injection time in the range of $10 \mathrm{fs} .{ }^{14}$ Rigid ring structures consisting of saturated $-\mathrm{C}-\mathrm{C}$ - bonds like cyclooc$\operatorname{tane}^{10}$ and adamantane $\mathrm{e}^{10,15}$ can be used as electronic tunneling barriers to slow down HET. Similar ring structures have been used earlier to slow down electron transfer in donor-bridge-acceptor molecules. ${ }^{16,17}$ The electronic interaction at the interface can be enhanced if a shorter bond is formed between the chromophore and the semiconductor than is realized by the $-\mathrm{COOH}$ anchor group. For example, the chromophore catechol binds directly via its two oxygen atoms to one or two $\mathrm{Ti}$ atoms on the $\mathrm{TiO}_{2}$ surface. $^{18,19}$ The thus formed molecular complex gives rise to optical transitions where the electron is lifted from the catechol ground state directly into the many empty electronic states on the $\mathrm{TiO}_{2}$ surface from where the electrons escape to bulk states of $\mathrm{TiO}_{2}{ }^{20}$ Direct optical charge transfer transitions have been studied extensively for donor-acceptor pairs in the gas phase and in solution, ${ }^{21}$ specifically also a molecular complex formed between three catechol moieties and one Ti-ion. ${ }^{22}$ Direct optical charge transfer is completely different from lightinduced HET since in the latter case on photon absorption the electron stays localized on the chromophore prior to the electron transfer step. For example, the first excited singlet state of perylene attached via the $-\mathrm{COOH}$ anchor group extends only very little onto the $\mathrm{TiO}_{2}$ surface. ${ }^{13}$ The yield of these two different electron injection mechanisms has been compared directly making use of a double chromophore consisting of perylene covalently linked to catechol and the latter chemisorbed on the $\mathrm{TiO}_{2}$ surface. It turned out that direct optical charge transfer from the ground state of the catechol moiety has much smaller oscillator strength than optical excitation of the first excited singlet state of perylene. ${ }^{23}$ Thus, light-induced HET is the more efficient process and consequently more attractive for device applications than direct optical charge transfer transitions.

Recently, two different mechanisms have been discussed for ultrafast light-induced HET. A nonadiabatic HET mechanism without restriction in the electronic coupling strength follows from a fully quantum mechanical model for both the electronic and the nuclear coordinates. ${ }^{24-27} \mathrm{~A}$ system of reduced dimensions is considered. In order to obtain quantitative predictions important parameters of this model have to be determined from a fit to experimental data. Alternatively, parameter values for the model can be obtained also from independent $a b$ initio calculations. ${ }^{13,28}$ On the other hand, an adiabatic HET mechanism has been derived from timedependent $a b$ initio DFT calculations ${ }^{29}$ where a parameter fit is not required. The TDDFT model is based, however, on a classical treatment of the nuclear coordinates. Hitherto, three different types of experimental data are available that can be compared with predictions of the theoretical models. Firstly, different ultrashort injection times of the Pe' chromophore that are controlled by different anchor-bridge groups, ${ }^{6,7}$ secondly, different line-widths in the linear absorption spectra of the $\mathrm{Pe}$ ' chromophore when attached to $\mathrm{TiO}_{2}$ via different anchor-bridge groups, ${ }^{12}$ Thirdly, different orientations of the Pe' chromophores when attached via different anchor groups to the rutile $\mathrm{TiO}_{2}(110)$ surface. ${ }^{30}$ In this paper, energy distribution curves for the injected electrons are compared with the predictions of different theoretical models for light-induced HET. ${ }^{24-27,29,31,32}$ The energy distribution curves for the injected electrons were obtained applying surface science techniques combined with optical spectroscopy, in particular femtosecond two-photon photoemission (2PPE) ${ }^{7}$

\section{Methods}

Details concerning sample preparation, Ultraviolet Photoemission Spectroscopy (UPS) and two-photon photoemission (2PPE) used to obtain the data for this work have been described already in our earlier papers. ${ }^{7,20}$ It is sufficient to summarize here some important points. Several different ultra-high vacuum (UHV) chambers, each equipped with a load-lock port, were employed for preparing and characterizing the samples. A mobile UHV chamber served as the shuttle for transporting the samples between the different UHV chambers, thereby maintaining ultrahigh-vacuum conditions. The bare $\mathrm{TiO}_{2}(110)$ crystal surface was cleaned via sputtering and annealing cycles. The perylene molecules with the different bridge-anchor groups were adsorbed from solution onto the $\mathrm{TiO}_{2}(110)$ surface. This preparation step was carried out in a specifically designed UHV chamber 
allowing for an easy switch between UHV conditions and exposure of the electrode to an inert gas atmosphere and then to a liquid solution. The perylene chromophores were always equipped with two lateral spacer groups, tertiary butyl, and the thus modified chromophore is labelled Pe'. The adsorption geometry of the $\mathrm{Pe}$ ' chromophores anchored with different groups on the rutile $\mathrm{TiO}_{2}(110)$ surface was determined from angle and polarization dependent 2PPE signals. ${ }^{30}$

The 2PPE signals were measured with femtosecond resolution applying a pump-probe technique which makes use of two ultrashort laser pulses. The pump and the probe pulse were shifted against each other on the time axis with the help of an optical delay stage. 2PPE was chosen as the pump-probe technique since it provides the necessary sensitivity for addressing femtosecond lifetimes in a molecular monolayer on a planar surface. The laser pulses entered the UHV chamber through a quartz window at an angle of $45^{\circ}$ with respect to the $\mathrm{TiO}_{2}(110)$ surface. The laser system was driven by a commercial Ti:sapphire oscillator followed by a $150 \mathrm{kHz}$ regenerative amplifier. Two non-collinear optical parametric amplifiers (NOPAs) generated ultrashort pulses and their frequency doubled output supplied photons with two different central wavelengths, i.e. $440 \mathrm{~nm}(2.82 \mathrm{eV})$ for the pump and $280 \mathrm{~nm}(4.43 \mathrm{eV})$ for the probe. ${ }^{33}$ The photoemitted electrons were detected with a time of flight spectrometer (TOF) where the arrival time is a measure of the kinetic energy of the respective photoemitted electrons. The instru-

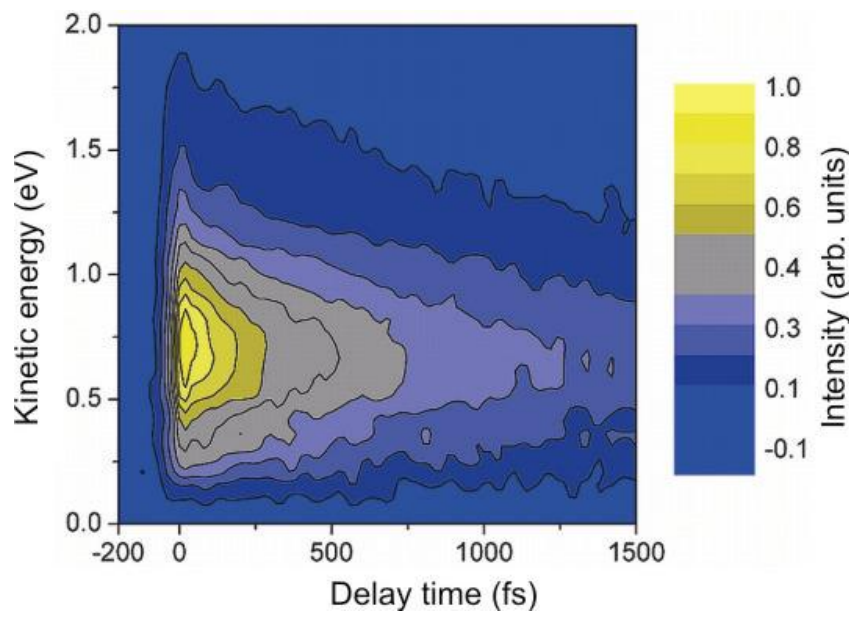

Figure 1. Pseudo 3D map of a 2PPE measurement with $\mathrm{Pe}$ ' achored via the propionic acid anchor-bridge group on the rutile $\mathrm{TiO}_{2}$ (110) surface. Details are explained in the text. mental cross-correlation function was obtained as a 2PPE signal measured on a polycrystalline gold surface where the intermediate state is known to have a short enough lifetime not to add to the width of the cross correlation function. The 2PPE signals collected on a given sample can be arranged in the form of a pseudo three-dimensional plot where different signal amplitudes are represented by plateaus of different gray shades (colours) and the two axes of the plot are: the time elapsed after a reference time (time zero) and the kinetic energy of the photoemitted electrons. A corresponding pseudo threedimensional plot is shown in figure 1 for $\mathrm{Pe}^{\text {' }}$ anchored via propionic acid on the rutile $\mathrm{TiO}_{2}(110)$ surface. It is clear from figure 1 that a cross section cut parallel to the time axis will yield the transient behaviour of the 2PPE signal whereas a cross section cut parallel to the energy axis will represent the energy distribution at the respective time where the cross section meets the time axis.

Linear absorption of adsorbed perylene chromophores was measured with the Bruker spectrometer IFS66v combined with an ultra-high-vacuum chamber for the sample. Details of the set-up have been described before. ${ }^{34}$ Compared to our earlier IR studies two things were changed to perform linear absorption measurements in the visible spectral range: the IR detector was replaced by a detector for visible light ( $\mathrm{Si}$ diode) and direct transmittance was measured in place of the ATR multi-pass arrangement for the earlier IR studies.

\section{Results and discussion}

\subsection{Vibrational structure in the linear absorption spectrum}

Figure 2 presents data points (open spheres) of the linear absorption spectrum for the Pe' chromophore attached via the propionic acid to the rutile $\mathrm{TiO}_{2}$ (110) surface. The data points were obtained as the difference of transmittance signals measured in the absence and presence of the chromophores. The drawn-out curve is a fit to the experimental data points, i.e. the curve is a Voigt spectrum consisting of a Gaussian of $145 \mathrm{meV}$ FWHM and a Lorentzian of $23 \mathrm{meV}$ FWHM. The latter value was fixed according to the measured HET time of $47 \mathrm{fs}$ for this system (see below). The halfwidth of the Gaussian was obtained from the best fit to the experimental data. 
The linear absorption spectrum of the same chromophore measured on a colloidal anatase layer of $\mathrm{TiO}_{2}{ }^{35}$ is shown for comparison (dash-dotted curve). The latter data was obtained as a smooth curve since the inner surface of the nano-structured anatase layer probed by the light beam was several hundred times larger than the surface area probed on the rutile single crystal. The linear absorption spectrum of the same perylene compound dissolved in a mixture of toluene and methanol $(1: 1)$ is also shown for comparison (dashed curve). The following conclusions can be drawn from the data shown in figure 2 . The linear absorption spectrum of perylene anchored via $-\left(\mathrm{CH}_{2}\right)_{2}-\mathrm{COOH}$ on the rutile $\mathrm{TiO}_{2}$ (110) surface showed one clear vibrational peak with the maximum slightly red-shifted from the maximum for the spectrum measured in the above solvent, ${ }^{35}$ it was slightly more red-shifted from the maximum measured on the $\mathrm{TiO}_{2}$ layer composed of anatase colloids. ${ }^{12}$ The width of the vibrational peak measured on rutile $\mathrm{TiO}_{2}(110)$ is dominated by inhomogeneous broadening considering the halfwidth for the Gaussian of $145 \mathrm{meV}$ compared to the half-width for the Lorentzian of $23 \mathrm{meV}$. We attribute this significant inhomogeneous broadening on the surface of the rutile single crystal to variations in the local environments to which the individual perylene chromophores in the ensemble are exposed. Figure 2 shows that the inhomogeneous broadening on the rutile surface is of the same order of magnitude as that on the anatase surface. Light-induced HET showed

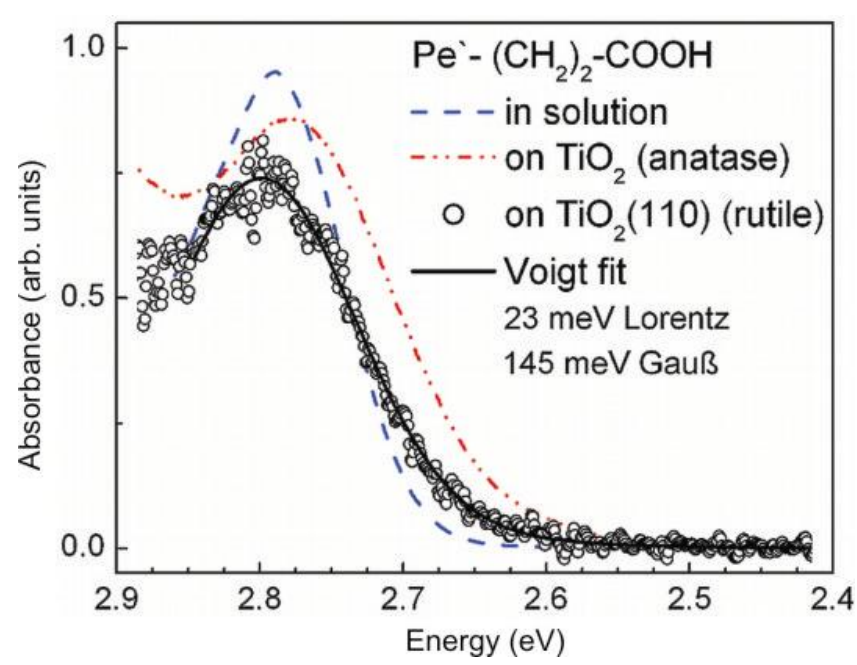

Figure 2. Linear absorption data (open spheres) for Pe' chromophores anchored via propionic acid on the rutile $\mathrm{TiO}_{2}$ (110) single crystal surface. Other information contained in this figure is explained in the text. very similar time constants on the two different stoichiometric $\mathrm{TiO}_{2}$ crystal surfaces, i.e. of anatase and rutile. ${ }^{7}$ Due to the $0.2 \mathrm{eV}$ larger band gap ${ }^{36}$ and the much thinner colloidal anatase sample compared to the thick rutile single crystal one can measure all the vibrational peaks of perylene on the surface of anatase $\mathrm{e}^{35}$ whereas only the first vibrational peak can be identified on the surface of rutile (figure 2). Fitting only one peak on rutile compared with four peaks on anatase is of course less reliable. Thus, we refrain from interpreting apparent numerical differences in the fit to the one peak measured on rutile compared to the four peaks measured on anatase. The important result in figure 2 is the occurrence of one clear vibrational peak in the linear absorption spectrum with a similar width as observed on anatase. The absorption peak due to the adsorbed perylene chromphores can be separated from the onset of the band to band transition in the rutile crystal only if the peak is not broader than in the above system. For example, with the $-\mathrm{COOH}$ anchor group instead of propionic acid the linebroadening of the Lorentzian is much stronger in agreement with the much shorter injection time of 9 fs (compare below) or $10 \mathrm{fs}^{6}$ for the latter compound so that the vibrational structure is lost in the absorption spectrum. ${ }^{12}$ It should be noted that a much stronger inhomogeneous broadening than observed in figure 2 could also wash out the vibrational structure in a corresponding experimental system.

\subsection{Photoionization spectrum for the ground state of perylene chromphores anchored on rutile $\mathrm{TiO}_{2}$ (110)}

With Pe' chromophores anchored on the rutile surface the UPS spectrum showed a new pronounced peak to the low energy side of the UPS spectrum of the rutile crystal. The peak arose from photoionization of the ground state of perylene. It is shown in figure 3 for Pe' anchored via propionic acid on the rutile $\mathrm{TiO}_{2}$ (110) surface. The photoionization energy for the ground state of the perylene chromophore near the $\mathrm{TiO}_{2}$ surface $(6.3 \mathrm{eV})$ is decreased by about $0.6 \mathrm{eV}$ compared to the photoionization energy of $6.9 \mathrm{eV}$ for the perylene chromophore in the gas phase. ${ }^{37}$ This shift is ascribed to screening by the $\pi$-electrons in the neighbouring perylene chromophores. Screening in a close packed 2-dimensional layer of aromatic molecules facilitates in most sys- 
tems a decrease in the ionization energy by about $1.5 \mathrm{eV}^{38}$ In the present system the shift is smaller due to the greater distance from the perylene neighbours which is brought about by the lateral spacer groups (tertiary butyl). There might be additional smaller contributions to the screening coming from the electrons in the adjacent $\mathrm{TiO}_{2}$ electrode and from the polarization of the $\mathrm{TiO}_{2}$ electrode which has an optical dielectric constant of about 7 and a static dielectric constant of $170 .^{39}$ The chemical nature of the anchor-bridge group has little influence on the shape of the ionization peak since the latter was similar with all the different anchor-bridge groups that we have investigated. The onset energy corresponding to the HOMO position in the band gap of $\mathrm{TiO}_{2}$ (compare below) was shifting by smaller amounts in the range of $0 \cdot 1-0 \cdot 2 \mathrm{eV}$ according to the specific anchor-bridge group employed. It should be noted that the work function of the rutile $\mathrm{TiO}_{2}(110)$ surface decreased on adsorption of the anchorbridge groups by 0.5 to $0.7 \mathrm{eV}$. The decrease in the work function was caused by a corresponding shift in the vacuum level. ${ }^{7}$ From electrochemical experiments it is well-known that there is a downward shift of the band edges of $\mathrm{TiO}_{2}$ with respect to the energy of a reference electrode when the acidity of the contact solution is increased. ${ }^{40}$

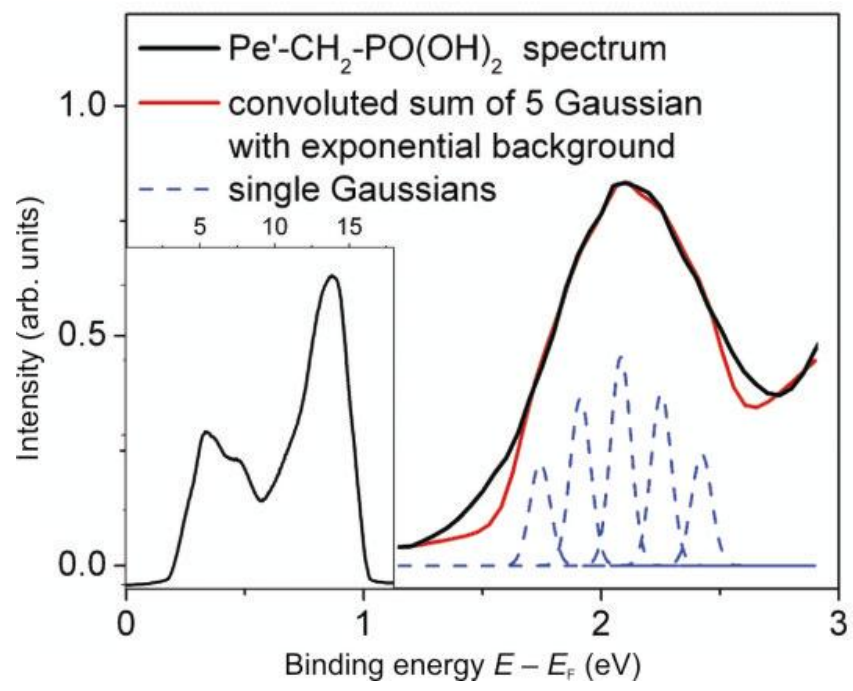

Figure 3. Ionization spectrum of $\mathrm{Pe}^{\prime}$ chromophores anchored via propionic acid on the rutile $\mathrm{TiO}_{2}$ (110) surface. The inset shows the complete ultraviolet photoelectron (UP) spectrum of the clean $\mathrm{TiO}_{2}$ (110) surface. The dashed curves show the decomposition into individual Gaussians and the thin smooth curve (red) the resultant fit to the peak including the exponential background. Further details are explained in the text.
The UP spectrum showed for the present systems in ultra-high vacuum that there was no noticeable band bending on adsorption of the anchor-bridge groups, i.e. the conduction band remained essentially flat near the surface. ${ }^{41}$ The ionization peak appearing at the red side of the UP spectrum (figure 3) of rutile $\mathrm{TiO}_{2}$ (110) due to $\mathrm{Pe}$ ' chromophores anchored via the propionic acid was fitted with 5 Gaussians. The peak positions of the individual peaks were placed at an energy distance of $0 \cdot 17 \mathrm{eV}$. The latter energy was chosen in accordance with the apparent vibrational structure of low resolution perylene spectra. ${ }^{12}$ Convoluting the spectrum composed of the individual peaks with the energy resolution of $80 \mathrm{meV}$ of our UPS apparatus and adding the exponential background that stems from the valence band of $\mathrm{TiO}_{2}$ resulted in a single apparent broad peak represented by the smooth (red) curve in figure 3 . There was a two-fold motivation for choosing this fit procedure. First, the photoionization spectrum of perylene in the gas phase shows vibrational structure with this energy spacing of $0.17 \mathrm{eV} .{ }^{37}$ Second, the linear absorption spectrum of perylene anchored on rutile (figure 2) and more obviously on anatase $\mathrm{TiO}_{2}$ showed vibrational structure with the same energy spacing. ${ }^{12}$ The fit shown in figure 3 illustrates the following physical picture of the photoionization process. Photons with excess energy with respect to the photoionization threshold generate the ionized state of perylene in a vibrationally excited state. The increasing apparent binding energy at the abcissa of figure 3 for consecutive individual Gaussians shown to the high energy side of the first Gaussian does not stem from photoionization of different electronic states with increasing binding energy but represents an increasing vibrational excitation for the ionized perylene chromophore on photoionization. The electronic energy of the ionized perylene in its vibrational ground state; HOMO, corresponds to the position of the $0-0$ transition, i.e. to the first Gaussian with the lowest photoionization energy in the spectrum. The photoionization spectrum of the anchored perylene chromophores differs from the absorption spectrum $^{12}$ and from the photoionization spectrum in the gas phase ${ }^{37}$ by a larger half-width for the individual Gaussian peaks and also in the energy where the maximum amplitude occurs. In the photoionization spectrum shown in figure 3 the peak with maximum amplitude corresponds to the $0-3$ and $0-4$ vibrational transitions whereas in the absorption spectrum $^{12}$ and in the photoionization spectrum in the 
gas phase ${ }^{37}$ the first peak attains the highest amplitude which corresponds to the $0-0$ transition. The shift of the maximum amplitude to a higher vibrational excitation of ionized perylene is ascribed to a larger value for the reorganization energy of perylene anchored on $\mathrm{TiO}_{2}$ compared to perylene in the gas phase. Placed near the $\mathrm{TiO}_{2}$ surface the perylene chromophore must undergo a larger change in the equilibrium nuclear coordinates on ionization than the isolated perylene chromophore in the gas phase. Also, the different shape of the absorption spectrum $^{12}$ shows that the shift in the nuclear equilibrium coordinates on the $\mathrm{TiO}_{2}$ surface must be much larger for ionized perylene than for perylene in the first excited singlet state. The interaction between the positive charge on the ionized perylene and the surface of $\mathrm{TiO}_{2}$ must be much stronger than that of the dipole moment in the first excited singlet state of perylene. We are not aware of any theoretical calculation that has addressed this behaviour for the present system but the effect is not surprising in view of the strong electronic and static polarizability of $\mathrm{TiO}_{2}{ }^{39}$ and the fact that the response to a change in the electric charge should be stronger than to a change in the dipole moment. The strong change occurring in the nuclear equilibrium coordinates on ionization of perylene near the $\mathrm{TiO}_{2}$ surface is underlined by the fact that a fifth vibrational peak with large amplitude is required for the fit in figure 3 in contrast to the much smaller amplitude of the corresponding fifth vibrational peak in the linear absorption spectrum of perylene anchored on $\mathrm{TiO}_{2}{ }^{12}$ The FWHM of $180 \mathrm{meV}$ required for the individual Gaussian indicates considerable inhomogeneous broadening on the surface of the rutile $\mathrm{TiO}_{2}$ single crystal even though the chromophores show a strong alignment with respect to the crystal axes and a clear orientation of the long axis of perylene with respect to the surface plane which is controlled by the bonds formed by the respective anchor group. ${ }^{30}$

\subsection{Time dependence of the injected electrons}

Figure 4 shows four different 2PPE transients that were obtained as cross sections cut parallel to the time axis through the respective 2PPE contour plots (compare figure 1). The cross sections are cut along the peak amplitude of the respective signal which is maintained along the center of the contour plot. The transients in figure 4 contain two different contributions with consecutive onsets on the time axis, i.e. the contribution (population) of the excited singlet state of the chromophore (dash-dotted red curves) and that of the electrons injected from this molecular donor state (dashed blue curves).
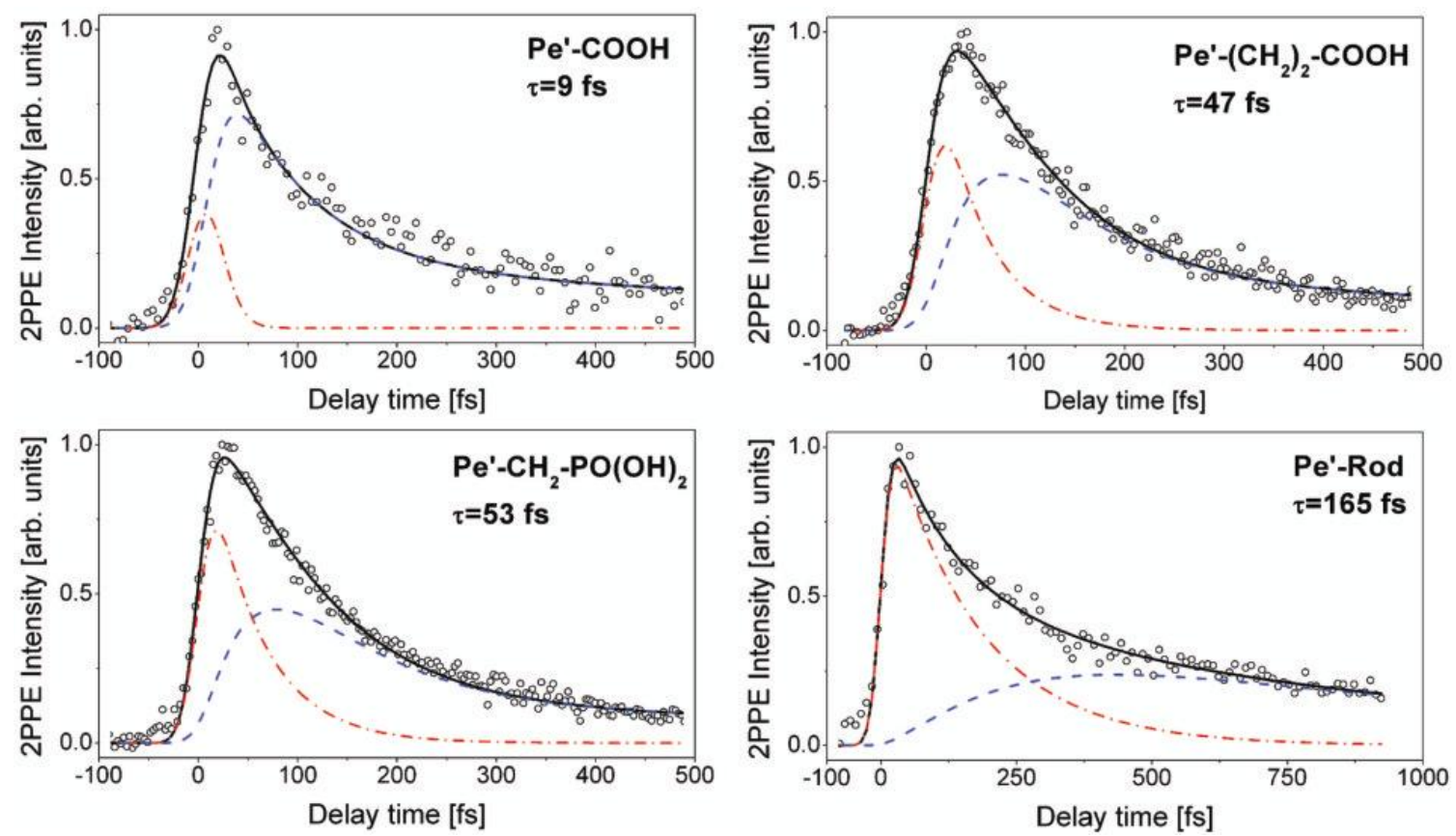

Figure 4. Transient 2PPE signals for Pe' chromophores anchored on $\mathrm{TiO}_{2}$ (110) with four different anchor-bridge groups given as the respective inset along with the respective injection time constant derived from a fit to the data. Details are explained in the text. 
To obtain a fit to the experimental 2PPE transient the time dependence of the reactant state (excited chromophore) can be modelled as a monoexponential decay and that of the product state (injected electron) correspondingly as a monoexponential rise, both of course with the same time constant and each contribution convoluted with the time response of the apparatus. Thus the obtained electron transfer time constants given as insets in figure 4 are in good agreement with the time constants obtained with the same Pe' compounds adsorbed on the surface of anatase colloids in a nano-structured layer, also measured in ultra-high vacuum. ${ }^{6,7}$ One can see smaller deviations from monoexponential behaviour in the transient absorption data, ${ }^{6}$ and there is some room for smaller deviations also in the fit to the 2PPE transients shown in figure 4 . Such deviations remain small, however, and it is not clear whether they are due to the fact that the measurements are probing an inhomogeneous ensemble or whether they are of a more principle nature. Deviations from monoexponential behaviour have been suggested by recent theoretical studies. ${ }^{28}$ Depending on the specific anchor-bridge group the injection time varied for the four cases displayed in figure 4 between $9 \mathrm{fs}$ and $165 \mathrm{fs}$. The injection time has been extended to $1 \mathrm{ps}$ when the $\mathrm{Pe}$ ' chromophore was attached to $\mathrm{TiO}_{2}$ via the so called tripod anchor-bridge group containing adamantane as an electronic tunneling barrier. ${ }^{10}$ It is evident from figures 1 and 4 that the energy distribution of the injected electrons can be obtained as a cross section cut parallel to the energy axis through the 2PPE contour plot and that the respective time has to be chosen such that only the injected electrons are addressed, i.e. at the time when the peak of the contribution of the injected electrons is occurring or later. It was checked in several $100 \mathrm{fs}$ long time windows that the energy distribution of the injected electrons remained virtually constant, i.e. there was no significant energy loss occurring for the electrons in the initial acceptor states on the surface prior to the electrons escaping into the bulk. ${ }^{7}$ Energy relaxation processes for the electrons in the primary acceptor states on the $\mathrm{TiO}_{2}$ surface with chemisorbed anchor groups turned out much slower than for hot electrons that were photogenerated in the form of electron-hole pairs in the bulk of $\mathrm{TiO}_{2}{ }^{7}$

\subsection{Energy distribution of the injected electrons and non-adiabatic mechanism of ultrafast light-induced HET}

The energy distribution of the injected electrons (figure 5) was obtained as a cross section cut
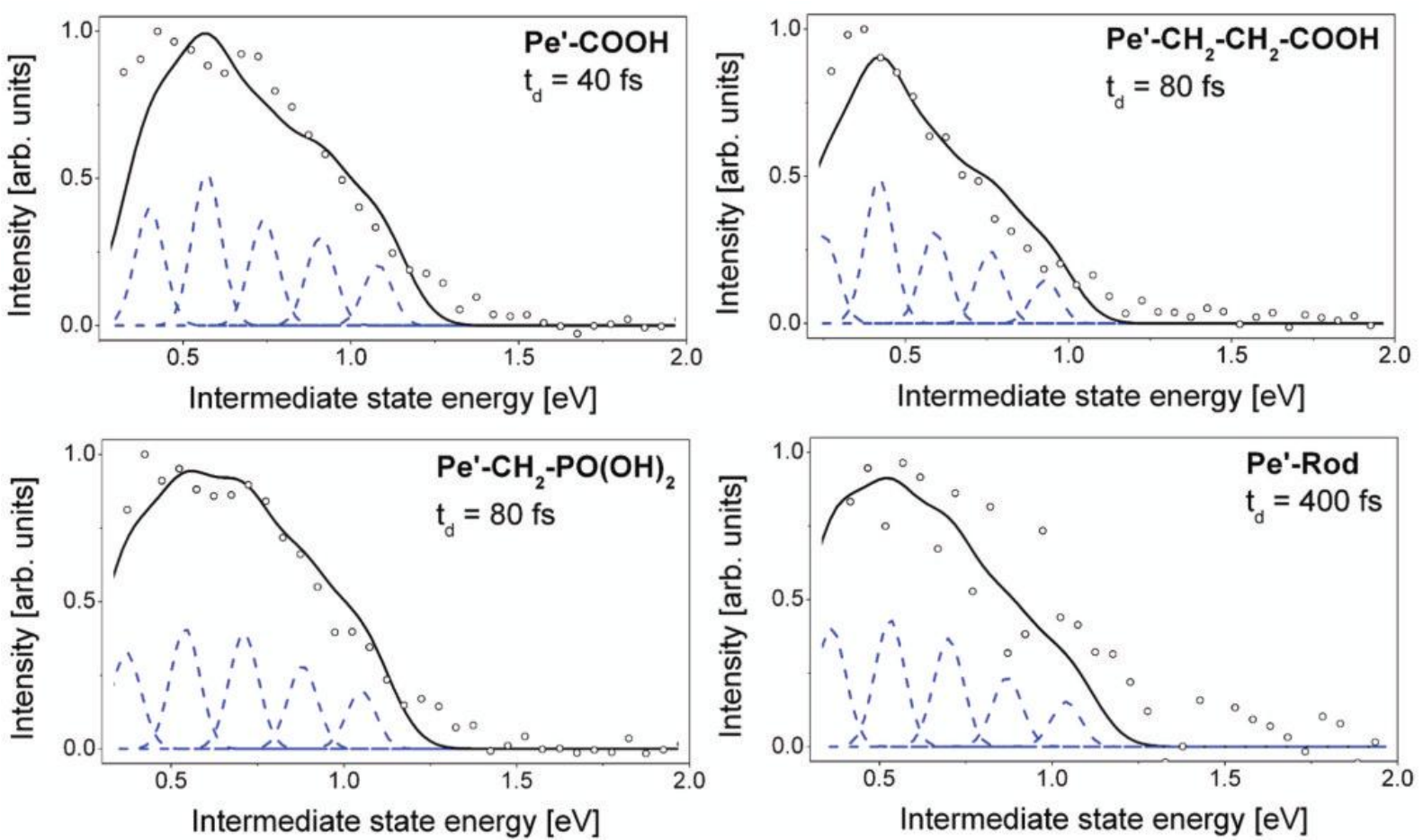

Figure 5. Energy distribution of the injected electrons for Pe' chromophores anchored with four different anchor-bridge groups (insets) on the rutile $\mathrm{TiO}_{2}(110)$ surface. Details are given in the text. 
parallel to the energy axis through the respective 2PPE contour plots (figure 1) taken at the time at which the contribution from the injected electrons to the 2PPE signal attained its maximum amplitude (compare figure 4).

It was found that the shape of the energy distribution curve was retained for at least $200 \mathrm{fs}$. A possible energy relaxation occurring on a longer time scale was difficult to detect since the amplitude of the 2PPE signal vanished due to the escape of the injected electrons into the bulk. The escape process brought the electrons out of the escape depth probed by the 2 PPE signal. ${ }^{20}$ There is considerable qualitative similarity between the energy distribution seen in the UPS spectrum, corresponding to photoionization of the ground state of the anchored chromophores (figure 2), and the 2PPE spectrum for the injected electrons (figure 5). In both cases the product state is a vibrationally excited ionized chromophore but in the first case the other product state is the electron in a vacuum state whereas in the second case the injected electron is located in an acceptor state on the surface of the semiconductor. ${ }^{7,24}$ The simplest assumption about the matrix element describing the electronic interaction between the excited molecular electronic donor state and empty electronic states of the semiconductor is a constant value over the whole energy range spanned by the conduction band. ${ }^{24,42} \mathrm{Ab}$ initio calculations have modified this simple physical picture, but not decisively. ${ }^{28}$

The width of the energy distribution for the injected electrons is controlled by the Franck-Condon factors for generating a vibrationally excited ionized chromophore. In the wide band limit this leads for only one dominant mode with energy $0.17 \mathrm{eV}$ to a corresponding energy width $n \times 0 \cdot 17 \mathrm{eV}$, where $\mathrm{n}$ is the highest vibrational excitation of the ionized chromophore corresponding to a finite value of the corresponding Franck-Condon factor. To fit the energy distribution in the 2PPE signal five Gaussians were required with energy spacing of $0.17 \mathrm{eV}$. The maximum amplitude appeared for the fourth peak. This decomposition is very similar to that of the UPS spectrum (figure 3). The Gaussian with the highest energy relative to the conduction band edge in the distribution curve for the injected electrons matches the energy of the Gaussian for the excited molecular donor level. The latter is obtained from the energy of the highest energy peak in the respective UPS spectrum, i.e. the HOMO level, by adding the photon energy absorbed by the anchored chromphore. Electron injection from the vibrational excited electronic molecular donor state inserts the electron into isoenergetic electronic acceptor states on the crystal surface and below. The specific case illustrated in figure 6 is electron injection from the vibrational ground state of the electronic excited molecular donor level, where isoenergetic electron transfer is illustrated by the highest black arrow pointing toward the semiconductor surface. Photoionization of the excited molecular donor state via electron injection gives rise also to a vibrationally excited ionized chromophore as the molecular product state. In the latter case the energy of the injected electron is lower by the respective energy required for vibrational excitation of the ionized chromophore. This is illustrated by the second highest black arrow pointing toward the semiconductor surface in figure 6. Correspondingly, higher vibrational excitation of the ionized chromophore by multiples of the dominant vibrational energy mode, i.e. by $n \times$ $0.17 \mathrm{eV}$ with $n=0,1,2,3,4$, lowers the energy of the injected electron by the respective amount as illustrated by the other black arrows in figure 6 . We address the ultrafast ET reactions as non-adiabatic based on this feature, i.e. electron injection into an

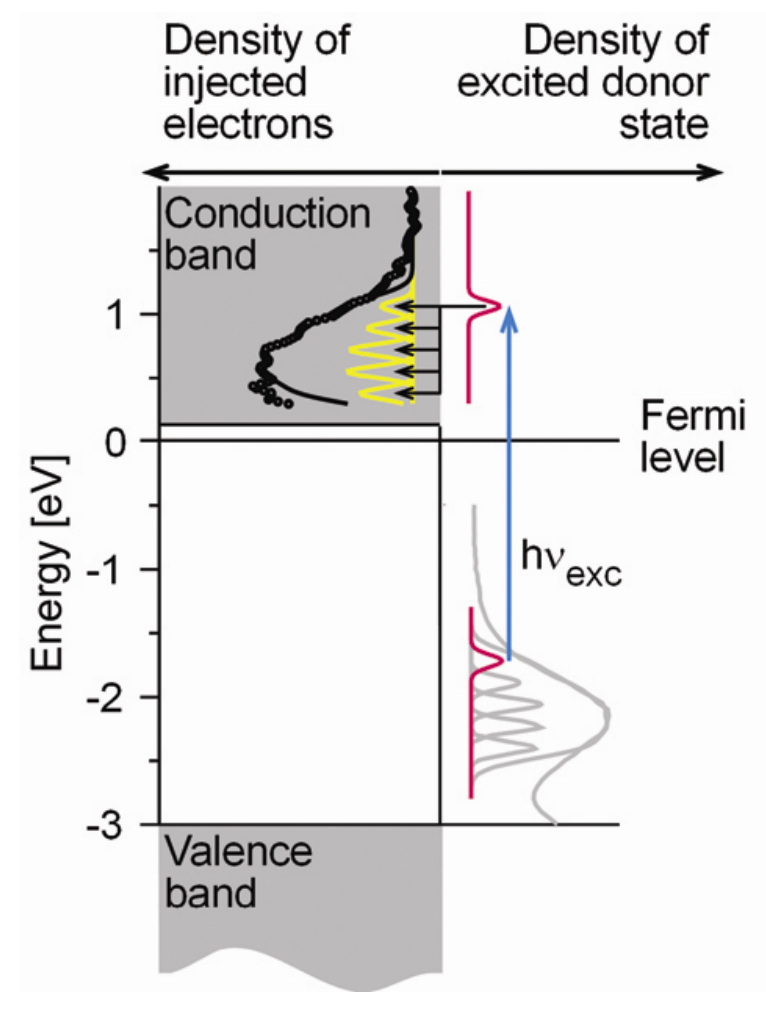

Figure 6. Illustration of the fully quantum mechanical injection model explained in the text. 
electronic level $680 \mathrm{meV}$ below the donor level compared to the electronic interaction energy of $13 \mathrm{meV}$ for this system.

It should be noted here that the fully quantum mechanical model ${ }^{24-27,42}$ retains all the Franck-Condon factors for vibrational excitation of high energy quantum vibrational modes in the ionized molecular product state irrespective of the strength of the electronic interaction. Previously, it was often assumed that the latter feature was restricted to weak electronic interaction since it was presented only in the framework of quantum mechanical perturbation treatments. The fully quantum mechanical model of ultrafast HET retains this feature of non-adiabatic electron transfer also for strong electronic coupling. In the fully quantum mechanical model of HET there is no automatic transition to adiabatic electron transfer for high electronic coupling strength and the excitation of high energy vibrational modes can not be ignored. This feature is in strong contrast to Gerischer's HET scenario ${ }^{1,31,51}$ and also to the Duncan-Prezhdo HET model ${ }^{29}$ which both ignore the excitation of high energy vibrational modes and consider only adiabatic HET pathways or nonadiabatic paths involving excitation of phonons with very small energy. Light-induced non-adiabatic HET is ultrafast, i.e. it occurs on a timescale of a few fs in the present system, provided the chromophore is attached via a sufficiently short anchor group, e.g. $-\mathrm{COOH}$ and the system fulfills the wide band limit. The different anchor-bridge groups entail smaller differences in the energy of the excited molecular donor level, i.e. in the energy of the first (highest energy) peak in the 2PPE spectrum for the injected electrons (figure 5). Those differences arise from corresponding differences in the HOMO levels for the perylene chromophore adsorbed via different anchor-bridge groups. Only the width of the Lorentzian contribution to an individual peak depends on the electron transfer time whereas the total width is dominated by the Franck-Condon progression (figure 4). Thus, the total width of the energy distribution is virtually independent of the strength of the electronic interaction between the excited molecular donor state and the electronic acceptor states on the semiconductor surface. The wide band limit is fulfilled in the present system ${ }^{42}$ if the electron can find a suitable electronic acceptor state on the semiconductor surface for every possible vibrational excitation of the ionized chromophore, i.e. all the possible Franck-Condon factors for vibrational excitation of the ionized chromophore can be realized. To fulfill the wide band limit the corresponding wide energy range of electronic acceptor states must be offered between the excited molecular donor level and the lower edge of the conduction band (figure 6). The measured energy distributions shown in figure 5 suggest a cut-off at the low energy side of the spectrum but the missing part of the spectrum is expected to be fairly small, so that the wide band limit ${ }^{42,43}$ is valid to a good approximation in these experimental systems. The injection scheme in figure 6 is a pictorial representation of the fully quantum mechanical model of photo-induced HET for both the electronic and the vibrational degrees of freedom. ${ }^{24,42}$ Note in figure 6 the energy width of the donor state is much smaller than that for the distribution of the injected electrons. Note also the energy shift between the centers of the two different distributions. Both these features are distinctly different from the predictions according to Gerischer's HET scenario ${ }^{1,31,51}$ and those of the Duncan-Prezhdo HET mode $^{29}$ which both allow essentially only for adiabatic electron transfer resulting in identical energy distributions curves for the molecular donor state and the injected electrons. Parameter values for the fully quantum mechanical model are obtained from a fit to experimental data ${ }^{12}$ or from independent calculations, e.g. DFT calculations. ${ }^{13,28}$ The behaviour of the system has also been calculated for situations where the wide band limit is not fulfilled. ${ }^{44}$ The main effect of moving the excited molecular donor level closer to the lower edge of the conduction band is the loss of the simple exponential decay behaviour which is then substituted by a more complex oscillation in the amplitude due to interferences and by non-exponential decay. Recently, the relationship between the measured 2PPE signal and the energy distribution of the injected electrons has been calculated using a tight binding model for the semiconductor. ${ }^{32}$ Our group has described experimental transients indicating an influence of vibrational wavepacket motion in the excited molecular donor state on the electron transfer process. ${ }^{45}$ Our more recent experiments, however, were carried out with better control over the preparation procedure for the experimental systems and with higher time resolution. They did not substantiate the above notion, neither in the transient absorption measurements ${ }^{6}$ nor in the 2PPE transients (figure 4). Rapid transfer of the injected electrons from the initially occupied surface states to bulk states of $\mathrm{TiO}_{2}$ has been measured ${ }^{20}$ and 
are in agreement with the predictions of sophisticated TDDFT model calculations. ${ }^{46-48}$ It should be noted that the above model of photo-induced HET as illustrated in figure 6 is essentially a non-adiabatic mechanism which can also accommodate the ultrafast time scale, ${ }^{42,24}$ e.g. the measured 9 fs electron transfer time for the perylene chromophore attached to $\mathrm{TiO}_{2}(110)$ via the $-\mathrm{COOH}$ anchor group (figure 4). ${ }^{6}$ Previously, for thermal ET reactions it had been assumed quite generally that non-adiabatic electron transfer is slower and adiabatic electron transfer is faster. ${ }^{49}$ It should be noted that for a given anchor group the same line-width broadening due to ultrafast electron transfer has been measured for all the vibrational peaks in the linear absorption spectrum of the $\mathrm{Pe}$ ' chromophore anchored on $\mathrm{TiO}_{2}{ }^{12}$ Thus, electron transfer occurs with the same time constant from all the different vibrationally excited levels of the molecular donor state. Intramolecular redistribution of vibrational excitation energy (ivr) is much slower for the perylene chromophore than ultrafast electron transfer if the chromophore is attached via a short anchor group to the surface of $\mathrm{TiO}_{2}$. In the case of perylene the time scale of ivr is picoseconds ${ }^{45}$ and that for HET is femtoseconds with a short anchor group (figure 4). For simplicity only electron transfer from the vibrational ground state of the excited molecular donor level has been illustrated in figure 6 for a moderately fast injection process corresponding to $\mathrm{Pe}$ '-propionic acid on $\mathrm{TiO}_{2}$. If the figure would be changed to show a Lorentzian line-width of $70 \mathrm{fs}$ in the Voigt profile characterizing the excited molecular donor state it would apply also to the fastest injection process of $9 \mathrm{fs}$ measured with $\mathrm{Pe}$ '- $\mathrm{COOH}$ (figure 4).

The most widely used scenario of HET in photoelectrochemistry has been introduced by Gerischer in $1972 .{ }^{31}$ It has remained popular until today. ${ }^{50}$ This scenario is often applied in the form of a pictorial representation of the energetics of HET with characteristic differences to figure 6 . At the time when the model was conceived reliable experimental data was not available for adsorbed dye molecules. The reorganization energy for dye molecules adsorbed on the surface of a semiconductor was not known and assumed in the range $0.4 \mathrm{eV}$. Since excitation of high energy vibrational modes (quantum modes) was not considered in Gerischer's injection model the latter predicted an energy distribution for the molecular donor state in the shape of a smooth Gaussian. ${ }^{31}$ The Gaussian energy distribution of the electronic excited donor state was considered the result of thermal fluctuations of small energy nuclear modes. ${ }^{31,51}$ Electron transfer was assumed essentially isoenergetic and thus the energy distribution of the injected electrons was virtually identical with that for the molecular excited donor state. In this respect Gerischer's scenario implied adiabatic HET, i.e. the energy distribution for the excited donor level is identical to that for the injected electrons. Until today the Gerischer scenario is frequently used in the photoelectrochemical literature. ${ }^{50}$ In principle an energy distribution curve in the form of a Gaussian is compatible also with the shape of the energy distribution curve for the injected electrons in a semiclassical non-adiabatic HET model. In the non-adiabatic mechanism, however, the energy distribution for the injected electrons is shifted to lower energies with respect to the energy distribution for the molecular donor state since the HET reaction involves reorganization energy due to the shift in nuclear equilibrium coordinates on photoionization of the molecular reactant. The shift and the width of the energy distribution (spectrum) for the injected electros are governed by two main factors. Firstly, the magnitude of the reorganization energy, and secondly the energy width covered by all the FranckCondon factors for excitation of vibrational modes (compare figure 6). In contrast, Gerischer's model assumes the energy distribution for the injected electrons isoenergetic with that for the molecular donor state. ${ }^{31}$ For an adiabatic HET mechanism the latter assumption should be fulfilled.

A profound theoretical justification of a Gerischertype scenario for the energy distribution of the excited adsorbed molecular donor state and for that of the injected electrons has recently been presented by Duncan and Prezhdo in the form of a TDDFT (time dependent density functional theory) model where the electronic interaction was calculated with an $a b$ initio approach and the role of the nuclear coordinates in the reaction scenario stemmed from a molecular dynamics calculation. ${ }^{29}$ Their calculations addressed a specific system, the sensitizer alizarin on $\mathrm{TiO}_{2}$, where the donor state lies close to the lower edge of the conduction band. The model calculations show that thermal fluctuations of small energy nuclear coordinates can indeed give rise to a broad inhomogeneous energy distribution for the molecular donor state, i.e. with $0.4 \mathrm{eV}$ FWHM at $350 \mathrm{~K}$ for the alizarin/ $/ \mathrm{TiO}_{2}$ system, as has been intuitively assumed by Gerischer. ${ }^{51}$ The energy dis- 
tribution of the injected electrons was found to be about the same energy width and essentially isoenergetic with that of the photoexcited molecular donor state. Note that this is in stark contrast to figure 6. A complication arises from the cut-off arising at the conduction band edge. The analytical fully quantum mechanical model incorporating a high energy vibrational mode predicts for the donor state lying close to the edge of the conduction band that the simple exponential decay behaviour valid in the wide band limit is lost here, instead there are oscillations in the population of the injected electrons due to interferences and non-exponential decay. ${ }^{44} \mathrm{We}$ would like to reiterate that the fully quantum mechanical injection model of reduced dimensions $^{24-27,42}$ gives a prediction very different from the Gerischer scenario in the wide band limit since the energy distribution of the injected electron is shifted to lower energies compared to the energy distribution of the excited molecular donor state and it is much wider. The latter prediction is in agreement with the experimental data measured with systems that fulfill the wide band limit. The results are illustrated in figure 6 . Note that the fully quantum mechanical non-adiabatic model is not restricted with respect to the strength of the electronic interaction, ${ }^{24-27,42}$ quite different from the previously customary perturbation treatment of non-adiabatic electron transfer. It can predict the experimental injection times of a few femtoseconds (figure 4$)^{5-9}$ in the wide band limit and also for the donor state lying close to the edge of the conduction band. ${ }^{24}$ For ultrafast HET according to the fully quantum mechanical model the energy width of the molecular donor state can be much smaller than the width of the energy distribution for the injected electrons as illustrated in figure 6. Recently, DFT calculations of the electronic interaction have been used for determining parameter values for a fully quantum mechanical injection model of reduced dimensions. ${ }^{28}$ Light-induced ultrafast HET at a metal electrode ${ }^{14}$ might even be more involved than at a semiconductor electrode since in addition to the excitation of a high energy vibrational mode in the molecular product state also non-adiabatic excitations of electrons in the metal electrode ${ }^{52}$ might play an important role. We would like to mention here that a fully quantum mechanical model of heterogeneous electron transfer without the restriction to weak electronic interaction has recently been described also for HET reactions in the dark. ${ }^{53}$ The width of the en- ergy distribution for the injected electrons shown in figure 6 stems from the vibrational progression due to excitation of a high energy $(0 \cdot 17 \mathrm{eV})$ vibrational mode (quantum mode) in the ionized perylene chromophore. The electron localized on the chromophore is strongly coupled to this high energy vibrational mode. Excitation of the latter is accompanied by electron injection into electronic acceptor states that lie far below the molecular donor state. This non-adiabatic feature of the fully quantum mechanical injection model can be pictured in analogy to the emission of a wide fluorescence spectrum from a narrow excited electronic state. The energy width of the generated spectrum, i.e. for the injected electrons and for the emitted photons, respectively, stems in both cases from the realization of all the Franck-Condon factors for exciting the high energy vibrational mode. In the case of HET this requires the validity of the wide band limit. ${ }^{42}$ The width of the energy distribution curve for the injected electrons depends insignificantly on the injection time, i.e. it is virtually independent of the strength of the electronic coupling (figures 4 and 5).

Consequences of the wide band limit ${ }^{43}$ are firstly exponential decay of the excited state population, provided the density of acceptor states does not vary extremely over energy, and secondly an electron transfer time that does not depend on the magnitude of the Franck-Condon factors even though the latter control the energy distribution of the injected electrons. ${ }^{24}$ The Franck-Condon factors are all realized in parallel and the sum over the Franck-Condon factors yields probability $1^{54}$ so that the electron transfer time is only controlled by the strength of the electronic coupling and the density of the electronic acceptor states. ${ }^{24}$ Accordingly, the electron injection time has been calculated for experimental systems fulfilling the wide band limit from the joint density of electronic states formed at the interface in the presence of the anchored chromophores. Applying Newn's chemisorption model ${ }^{43}$ to the width of the electronic donor level of the adsorbed chromophore the injection time has been deduced from the joint density of states. ${ }^{13}$ We have mentioned already that the above simplification does not apply if the molecular donor level lies only slightly above the conduction band edge, e.g. by only the energy width corresponding to the strength of the electronic coupling. For the conduction band to accommodate the whole spectrum of the injected electrons the electronic donor level must lie sufficiently high (figure 6). 


\section{Conclusions}

Our above interpretation of the measured 2PPE spectra, i.e. injection of electrons into the electronic acceptor states on the surface of the semiconductor (figure 5), and also of the UPS spectra, i.e. ionization of the ground state of the perylene chromophores anchored on $\mathrm{TiO}_{2}$ (figure 3), is based on the known vibrational structure in the ionization spectrum of perylene in the gas phase $\mathrm{e}^{37}$ and on the measured vibrational structure in the linear absorption spectrum of the perylene chromophores when attached via anchor-bridge groups to the surface of $\mathrm{TiO}_{2}$ (figure 2). ${ }^{12}$ Our interpretation of these spectra assumes that these seemingly smooth spectra are composed of individual vibrational peaks arising from the excitation of a dominant $0.17 \mathrm{eV}$ vibrational mode of the perylene chromophore. The seemingly smooth spectra are the result of inhomogeneous broadening that is even stronger than measured for the linear absorption spectrum of the chromophores anchored on the surface of $\mathrm{TiO}_{2}$ (figure 2).

The experimental data can be explained completely with the fully quantum mechanical model where electron transfer is non-adiabatic irrespective of the strength of the electronic coupling. ${ }^{24-28,42}$ One might argue that our experimental data do not refute conclusively the alternative interpretation described above as the Duncan/Prezhdo model ${ }^{29}$ which underscores the Gerischer scenario. ${ }^{31}$ Adiabatic or close to adiabatic electron transfer is considered in the latter two models and the excitation of high energy vibrational modes, e.g. the $0.17 \mathrm{eV}$ skeletal stretch mode of perylene, in the HET reaction and the corresponding influence on the spectra is ignored. Following the Gerischer scenario one could argue that the considerable width of the smooth room temperature spectra, UPS for the molecular ground state (figure 3) and 2PPE for the injected electrons (figure 5), could be the result of thermal fluctuations of classical nuclear coordinates leading at least at room temperature to an enormous broadening of the respective molecular states, as substantiated by the Duncan/ Prezhdo calculations for the excited molecular state. ${ }^{29}$ Making the latter assumption the energy width measured for the injected electrons (figure 5) would have to be identified with the energy width for the molecular donor state. This assumption is refuted, however, by the much narrower energy width for the donor state compared to the energy width for the injected electrons (compare the peak width in figure 2 with that in figure 5). Moreover, there is an energy shift between the two different energy distributions which follows automatically from nonadiabatic electron transfer described by the fully quantum mechanical model (figure 6) but can not be explained making the assumption of adiabatic or close to adiabatic HET as in the Gerischer scenario or the Duncan/Prezhdo HET model. ${ }^{29}$ Fitting the broad spectra for the injected electrons with the vibrational progression due to excitation of the $0.17 \mathrm{eV}$ vibrational skeletal stretch mode of perylene appears at hand since the same vibrational mode shows up prominently as vibrational structure in the photoionization spectrum in the gas phase ${ }^{37}$ and also in the linear absorption spectrum of the perylene chromophores anchored on $\mathrm{TiO}_{2}$ (figure 2). ${ }^{12}$ In our opinion the interpretation of the experimental spectra within the non-adiabatic fully quantum mechanical analytical model for light-induced ultrafast HET, considering explicitly strong coupling to the $0 \cdot 17 \mathrm{eV}$ skeletal stretch mode of perylene, is the valid interpretation of the measured spectra. In summary, we suggest here that light-induced HET from the aromatic chromophore perylene, anchored on the surface of the semiconductor $\mathrm{TiO}_{2}$ via a short molecular group, e.g. $-\mathrm{COOH}$, proceeds in the wide band limit as an ultrafast non-adiabatic electron transfer reaction.

A final definitive experimental proof for the nonadiabatic mechanism of ultrafast light-induced HET $^{24-28,42}$ can be obtained from measurements of the above shown spectra (figures 2 and 5) at low enough temperatures where the small energy classical nuclear modes are frozen so that these oscillators can not produce a wide energy distribution for the excited molecular state. Thus, the Duncan/Prezhdo model predicts that the energy distribution curve for the excited molecular donor state and correspondingly that for the injected electrons should become significantly narrower at low temperatures. ${ }^{29}$ In contrast, the total energy width controlled by the vibrational progression for the excitation of a high energy vibrational mode should not depend significantly on temperature, i.e. the non-adiabatic fully quantum mechanical model of HET would not predict a significant narrowing of the total width (envelope) for the energy distribution curve of the injected electrons at low temperatures (figure 5).

\section{Acknowledgements}

The authors are grateful to the German Science Foundation for financial support and to the Hahn 
Meitner Institut where experimental work was carried out.

\section{References}

1. Gerischer H and Willig F 1976 In Topics in current chemistry (ed.) F Boschke (Berlin: Springer) vol 61, p. 31

2. James $\mathrm{T} H 1977$ The theory of the photographic process (ed.) T H James (New York: Macmillan) 4th edn

3. O'Regan B and Grätzel M 1991 Nature 353737

4. Burfeindt $B$, Hannappel $T$, Storck $W$ and Willig $F$ 1996 J. Phys. Chem. 100 16463; The electron transfer time reported here was found later to depend on details of the preparation procedure. The spread in the values caused by unintentionally different preparation procedures was summarized as $(40 \pm 25) \mathrm{fs}$ in our following paper: Burfeindt B, Zimmermann C, Ramakrishna S, Hannappel T, Meissner B, Storck W and Willig F 1999 Z. Physikal. Chemie 212 67. Later our group had learned to reproduce the preparation procedure giving the shortest time constant for a given system, and we consider this preparation the best. Compare the HET time constant of $53 \mathrm{fs}$ for the same $\mathrm{Pe}-\mathrm{CH}_{2}-\mathrm{CH}_{2}-\mathrm{COOH}$ acid anchored on the rutile $\mathrm{TiO}_{2}$ (110) surface derived from the 2PPE transients (figure 4 of this paper)

5. Hannappel T, Burfeindt B, Storck W and Willig F 1997 J. Phys. Chem. B101 6799

6. Ernstorfer R, Gundlach L, Felber S, Storck W, Eichberger R and Willig F 2006 J. Phys. Chem. B110 25383

7. Gundlach L, Ernstorfer R and Willig F 2007 Progress in Surface Science $\mathbf{8 2} 355$

8. Huber R, Moser J, Grätzel M and Wachtveitl J 2002 J. Phys. Chem. 1066494

9. Benkö G, Kallioinen J, Korpi-Tommola J, Yartsev A and Sundström V 2002 J. Am. Chem. Soc. 124489

10. Gundlach L, Ernstorfer R and Willig F 2007 J. Phys. Chem. C111 13586

11. Guo J, She C and Lian T 2007 J. Phys. Chem. C111 8979

12. Wang L, May V, Ernstorfer R and Willig F $2005 \mathrm{~J}$. Phys. Chem. B109 9589

13. Persson P, Lundqvist M J, Ernstorfer R, Goddard III W A and Willig F 2006 J. Chem. Theory Comput. 2 441

14. Gundlach L and Willig F 2007 Chem. Phys. Lett. 449 82

15. Gundlach L, Felber S, Storck W, Galoppini E, Wei Q and Willig F 2005 Res. Chem. Intermed. 3139

16. Paddon-Row M N, Oliver A M, Warman J M, Smit $\mathrm{K} \mathrm{J}$, de Haas H O and Verhoeven J W $1988 \mathrm{~J}$. Phys. Chem. 926958

17. Koeberg M, de Groot M, Verhoeven J W, Lokan N R, Shephard M J and Paddon-Row M N 2001 J. Phys. Chem. A105 3417

18. Moser J, Punchihewa S, Infelta P P and Grätzel M 1991 Langmuir 73012
19. Duncan W R and Prezhdo O V 2005 J. Phys. Chem. 109365

20. Gundlach L, Ernstorfer R and Willig F 2006 Phys. Rev. B74 035324

21. Mulliken R S and Person W B 1969 Molecular complexes (New York: Wiley)

22. Borgias B A, Cooper S R, Koh Y B and Raymond K N 1984 Inorg. Chem. 231009

23. Wang L, Willig F and May V 2007 J. Chem. Phys. 126134110

24. Wang L, May V, Ernstorfer R, Gundlach L and Willig F 2007 In Analysis and control of ultrafast photo-induced reactions (eds) $\mathrm{O}$ Kühn and L Wöste (Berlin: Springer) vol 87

25. Wang L, Willig F and May V 2006 J. Chem. Phys. 124014712

26. Sebastian K L and Tachya M 2006 J. Chem. Phys. 124064713

27. Mohr J, Schmickler W and Badiali J P 2006 Chem. Phys. 324140

28. Li J, Nilsing M, Kondov I, Wang H, Persson P, Lunell S and Thoss M 2008 J. Phys. Chem. C112 12326

29. Duncan W R and Prezhdo O V $2008 \mathrm{~J}$. Am. Chem. Soc. 1309756

30. Gundlach L, Szarko J, Socaciu-Siebert L D, Neubauer A, Ernstorfer R and Willig F 2007 Phys. Rev. B75 125320

31. Gerischer H 1972 Photochem. Photobiol. 16 243; The model for the energy distribution of the electronic excited molecular donor state $D_{\text {donor }} *$ in this paper is the extension of an earlier model for electron injection in the dark where the corresponding occupied molecular donor state was labeled $D_{\text {red. }}$ Gerischer $\mathrm{H}$ 1961 Z. Physikal. Chem. (Neue Folge) 2749

32. Tsivlin D V, Willig F and May V 2008 Phys. Rev. B77 035319

33. Gundlach L, Ernstorfer R and Willig F 2007 Appl. Phys. A88 481

34. Letzig T, Schimper H-J, Hannappel T and Willig F 2005 Phys. Rev. B71 033308

35. Ernstorfer R 2004 Spectroscopic investigation of photoinduced heterogeneous electron transfer $\mathrm{Ph} \mathrm{D}$ Thesis (Berlin: Freie Universität)

36. Pascual J, Camassel J and Mathieu H 1978 Phys. Rev. B18 5606; Gupta V P and Ravindra N M $1980 \mathrm{~J}$. Phys. Chem. Solids 41591

37. Boschi R, Murrell J N and Schmidt W 1972 Faraday Discuss. Chem. Soc. $\mathbf{5 4} 116$

38. Pope M and Swenberg C E 1999 Electronic processes in organic crystals and polymers (New York: Oxford University Press) 2nd edn

39. Cronemeyer D C 1951 MIT Laboratory for insulation research Rept. 46; Parker R A 1961 Phys. Rev. 124 1719

40. Dutoit E C, Cardon F and Gomez W P 1976 Ber. Bunsenges. Phys. Chem. 80475

41. Gundlach L 2005 Surface electron transfer dynamics in the presence of organic chromophores $\mathrm{Ph} \mathrm{D}$ Thesis (Berlin: Freie Universität)

42. Ramakrishna S, Willig F and May V 2000 Phys. Rev. B62 R16330 
43. Muscat J P and Newns D M 1978 Progress in Surface Science 91

44. Ramakrishna S, Willig F, May V and Knorr A 2003 J. Phys. Chem. B107 607

45. Zimmermann C, Willig F, Ramakrishna S, Burfeindt B, Pettinger B, Eichberger R and Storck W $2001 \mathrm{~J}$. Phys. Chem. B105 9245

46. Rego L G C and Batista V S $2003 \mathrm{~J}$. Am. Chem. Soc. 1257989

47. Abuabara S G, Rego L G C and Batista V S $2005 \mathrm{~J}$. Am. Chem. Soc. 12718234

48. Duncan W R, Stier W M and Prezhdo O V $2005 \mathrm{~J}$. Am. Chem. Soc. 1277941
49. Marcus R A 1964 Annu. Rev. Phys. Chem. 15 155

50. Memming R 2001 Semiconductor electrochemistry (New York: Wiley)

51. Page 40 in ref 1 , page 245 in ref 30 , handwritten manuscript of $\mathrm{H}$ Gerischer given to $\mathrm{F} W$

52. Shenvi N, Cheng H and Tully J C 2006 Phys. Rev. A74 062902

53. Mohr J-H and Schmickler W 2000 Phys. Rev. Lett. 84 1051

54. Miller R J D, McLendon G, Nozik A, Schmickler W and Willig F 1995 In Surface electron transfer processes (New York: Wiley-VCH) ch. 5, p. 167 